\title{
Zbtb7 suppresses the expression of CDK2 and E2F4 in liver cancer cells: Implications for the role of Zbtb7 in cell cycle regulation
}

\author{
XUEFENG YANG ${ }^{2}$, XUYU ZU ${ }^{1,3}$, JING TANG $^{2}$, WEI XIONG ${ }^{2}$, \\ YUAN ZHANG ${ }^{2}$, FENG LIU ${ }^{3}$ and YUYANG JIANG ${ }^{3,4}$ \\ ${ }^{1}$ Clinical Medicine Institution, The First Affiliated Hospital; ${ }^{2}$ Nanhuan Hospital, University of South China, Hengyang, \\ Hunan 421001; ${ }^{3}$ The Key Laboratory of Chemical Biology, Guangdong Province, Graduate School at Shenzhen, \\ Tsinghua University, Shenzhen, Guangdong 518055; ${ }^{4}$ School of Medicine, Tsinghua University, Beijing 100084, P.R. China
}

Received December 13, 2011; Accepted March 19, 2012

DOI: $10.3892 / \mathrm{mmr} .2012 .846$

\begin{abstract}
Zbtb7, a member of the POK protein family, is involved in tumorigenesis and cellular differentiation by acting as a crucial transcription factor, but its role in cell cycle modulation remains uncharacterized. In the present study, CDK2 and E2F4, two cell cycle regulators, are shown to be downregulated at the mRNA and protein levels by Zbtb7 in HepG2 and QGY7703 cells. Moreover, we demonstrate that the activities of CDK2 and E2F4 promoters were suppressed by the modulation of $\mathrm{Zbtb} 7$ levels and that $\mathrm{Zbtb} 7$ represses promoter activities through a mechanism involving direct binding of Zbtb7 to the promoters. Furthermore, it was identified that the site at -259 to -252 within the CDK2 promoter is responsible for $\mathrm{Zbtb} 7$-induced repression of the promoter activity. It was found that siRNA-induced knockdown of Zbtb7 resulted in the suppression of cell cycle progression in HepG2 and QGY7703 cells. Collectively, these data indicate that CDK2 and E2F4 are the downstream targets of Zbtb7, and Zbtb7 may be a cell cycle modulator by regulating the expression of cell cycle-associated genes in liver cancer cells.
\end{abstract}

\section{Introduction}

Zbtb7, also known as LRF10, OCZF11 and FBI-1 (1-3), is a POK protein family member originally isolated as a cellular

Correspondence to: Dr Yuyang Jiang, The Key Laboratory of Chemical Biology, Guangdong Province, Graduate School at Shenzhen, Tsinghua University, Shenzhen, Guangdong 518055, P.R. China

E-mail: jiangyy@sz.tsinghua.edu.cn

Dr Xuyu Zu, Clinical Medicine Institution, The First Affiliated Hospital, University of South China, Hengyang, Hunan 421001, P.R. China

E-mail: zuxuyu0108@hotmail.com

Key words: Zbtb7, CDK2, E2F4, cell cycle regulation, promoter activity factor that binds to the inducer of short transcripts of human immunodeficiency virus-1 and the proximal GC-rich sequence of $A D H 5 / F D H(4,5)$. Zbtb7 has a critical function in cellular differentiation and its aberrant expression has been shown to be implicated in tumorigenesis in various organs including the lung, liver, spleen and thymus $(2,6)$. The mechanism by which Zbtb7 causes tumorigenesis is partly due to the repression of the tumor-suppressor gene ARF induced by Zbtb7 in mouse models (2). It has also been revealed that Zbtb7 plays a vital role in the late stage of osteoclastogenesis (3) and that Zbtb7 is involved in instructing early lymphoid progenitors in mice to develop into $\mathrm{B}$ lineage cells by repressing $\mathrm{T}$ cellinstructive signals produced by the cell-fate signal protein, Notch (7). Zbtb7 was found to be a modulator of a series of genes including Egr-1, c-Krox, cyclinA, E2F4, ADH5, Rb, FANS, p107 and ARF (2,5,8-11), suggesting a significant role for $\mathrm{Zbtb} 7$ in a range of biological activities.

Cyclin-dependent kinase 2 (CDK2) is thought to be a major cell cycle regulator functioning during the G1/S transition and $\mathrm{S}$ phase, and CDK activity is largely regulated positively through association with the appropriate cyclins and negatively by CDK inhibitors. CDK2 expression has been shown to be regulated at the transcription level by MITF in melanoma and the sequence spanning -100 to +108 was found to be sufficient for the basal activity of CDK2 promoter in NIH3T3 cells (12).

Since both Zbtb7 and CDK2 are involved in tumorigenesis and potential cis elements for Zbtb7 have been found within the promoter of CDK2, we investigated whether Zbtb7 regulates the expression of CDK2 in liver cancer cells. We demonstrated that this is indeed the case, using real-time PCR measurements of CDK2 mRNA and western blot analysis of CDK2 protein upon modulation of the $\mathrm{Zbtb} 7$ expression level, chromatin immunoprecipitations (ChIPs) and luciferase reporter assays.

\section{Materials and methods}

Cell lines and cell culture. Human liver cancer HepG-2 cells and QGY7703 cells (American Type Culture Collection, Manassas, VA, USA) were cultured in DMEM medium containing $10 \%$ 
fetal bovine serum (Hyclone, USA), L-glutamine ( $2 \mathrm{mM})$, streptomycin $(0.1 \mathrm{mg} / \mathrm{ml})$ and penicillin $(100 \mathrm{U} / \mathrm{ml})$ at $37^{\circ} \mathrm{C}$ in a humidified incubator supplied with $5 \% \mathrm{CO}_{2}$.

Plasmid preparation. Various 5'-3' deletion constructs of DNA fragments relative to the translation start site (ATG) were generated from HepG-2 cells. For construction of the putative CDK2 promoter deletion constructs, the reverse primer for PCR was GAAAAGCTTGAAGTTCTCCATGAA, and the forward primers were 5'-GAAAGATCTAATTTAACCTTAG GTCGATA-3' (528 bp) and 5'-GAAAGATCTCAAATGAAG AAGGCAT-3' (1046 bp). For the construction of the putative $\mathrm{E} 2 \mathrm{~F} 4$ promoter deletion constructs, the reverse primer for $\mathrm{PCR}$ was 5'-GCAAAGCTTACTTGGTGGTGAGCAGTC-3', and the forward primers were 5'-GCAAGATCTGTCTTATTCGG AGGCTGGAC-3' (301 bp), 5'-GCAAGATCTCAGTAGGGC AGCCTTTAG-3' (600 bp) and 5'-GCAAGATCTTATAGTG AGACCCACATCTC-3' (1152 bp). PCR-amplified products were digested with enzymes $B g l \mathrm{II}$ and HindIII, and cloned into the corresponding sites of the pGl4.10-basic firefly luciferase expression plasmid (Promega). The internal control pRL-TK Renilla Luciferase vector was purchased from Promega Corp. The Zbtb7 expression construct was generated by inserting a cDNA fragment obtained by reverse transcription PCR from HepG-2 cells into the pcDNA3.1 (-) vector at the HindIII sites. The PCR oligonucleotide primer pair used for the Zbtb7 cDNA amplification was as follows: 5'-CTTAAG CTTGCCACCATGGCCGGCGGCGTGG-3' and 5'-GTC AAGCTTTTAGGCGAGTCCGGCTGTGAAGTTAC-3'. All constructs were verified by sequencing.

Site-directed mutagenesis. Zbtb7 putative recognition site mutations of the CDK2 promoter were obtained by preparing the PMS1, PMS2, PMS3 and PDS2 constructs, using mutated primers. The plasmids with the mutation were generated by using a site-directed mutagenesis system (Promega) according to the manufacturer's protocol and pLuc528 construct was used as a template. The oligonucleotides used for mutagenesis (mutations indicated in bold italics) are as follows: GCCT $\boldsymbol{G} \boldsymbol{G} \boldsymbol{G} \boldsymbol{C} \boldsymbol{G} \boldsymbol{G}$ GATCATTTGC (PMS1 -329 to $-322 \mathrm{bp}$ ), GGCCT $\boldsymbol{G} \boldsymbol{G} \boldsymbol{G} \boldsymbol{G} \boldsymbol{G} \boldsymbol{C} \boldsymbol{G} \boldsymbol{G A G C C A G G G}$ (PMS2 -259 to $-252 \mathrm{bp}$ ), AGCAGGGGCGGGGCCTCTGGT (PMS3 -227 to -218 bp), GGCCT-------AGCCAGGG (PDS2 -259 to -252 bp). DNA sequencing was performed to confirm that the sequence of the PCR products was correct as compared with the CDK2 promoter published in the Human Genome database.

Transient transfections and luciferase assay. HepG-2 and QGY7703 cells $\left(1 \times 10^{5}\right)$ were seeded into 24-well plates, and $2 \mu \mathrm{l}$ of Lipofectamine-2000 (Invitrogen, Carlsbad, CA, USA) was used for plasmid transfection. At $48 \mathrm{~h}$ following transfection, the cells were harvested, lysed and luciferase activities were measured in triplicate using Dual Luciferase ${ }^{\mathrm{TM}}$ Reporter Assay System (Promega, Wallisellen, Switzerland).

Chromatin immunoprecipitation (ChIP) assay. ChIP assay was carried out to analyze the physical interaction between Zbtb7 and the CDK2 promoter in the HepG-2 cells. Formaldehyde was added at $1 \%$ to the culture medium for $10 \mathrm{~min}$ at $37^{\circ} \mathrm{C}$ to cross-link protein to DNA. Cells were washed twice with ice-cold 1X PBS, scraped and resuspended in ChIP sonication buffer (1\% Triton-100, 0.1\% deoxycholate, $50 \mathrm{mM}$ Tris 8.1, $150 \mathrm{mM} \mathrm{NaCl}, 5 \mathrm{mM}$ EDTA) containing protease inhibitors. The cells were sonicated to shear DNA to lengths between 500 and $2000 \mathrm{bp}$. The sonicated supernatant was diluted with ChIP dilution buffer, and incubated with antibody against Zbtb7 (Abcam) overnight at $4^{\circ} \mathrm{C}$ with rotation. The chromatin-antibody complexes were collected by Dynabeads Protein G (Invitrogen). Following three washes with pH 5.0 TE buffer (10 mM Tris, $1 \mathrm{mM}$ EDTA), the pellet was dissolved with $\mathrm{pH} 3.0$ TE buffer. Following precipitation with ethanol, the pellets were resuspended and treated with proteinase $\mathrm{K}$. The supernatant was extracted with saturated $\mathrm{NaCl}$ and precipitated with 2-propanol to recover the DNA. The primers for ChIP PCR were designed to amplify potential Zbtb7 binding sites in the CDK2 and E2F4 core promoter sequences. The primers used were as follows: 5'-GAAAGATCT AATTTAACCTTAGGTCGATA-3', 5'-GAAAAGCTTGAA GTTCTCCATGAA-3' (CDK2); 5'-GCAAGATCTCAGTA GGGCAGCCTTTAG-3, 5'-GCAAAGCTTACTTGGTGG TGAGCAGTC-3' (E2F4).

siRNA transfection. Cells were transfected with Zbtb7targeting siRNAs (5'-GCUGGACCUUGUAGAUCAAtt-3', 5'-UUGAUCUACAAGGUCCAGCtt-3') using a modified Oligofectamine protocol (Invitrogen). In brief, 1 day prior to transfection, cells were seeded without antibiotics to a density of 50-60\%. In all experiments, cells were transfected with siRNAs at a concentration of $50 \mathrm{nM}$. Control cells were treated with scramble RNA. Cells were harvested $48 \mathrm{~h}$ following siRNA treatment for cell cycle analysis and RT-PCR.

Real-time PCR analysis. Total RNA was prepared from cells using TRIzol reagent (Invitrogen Corp.) according to the manufacturer's instructions. Following isolation, RNA was reversely transcribed to cDNA using random primers (Promega). The reaction mixture containing SYBR Green PCR Master Mix (Takara) was run in a 7500 Real-Time PCR system (Applied Biosystems). The PCR primers used for quantitative RT-PCR were: 5'-ATTCATGGATGCCTCTGC-3', 5'-GCT CCGTCCATCTTCATC-3' (CDK2); 5'-CATCCAGTGGAA GGGTGTG-3', 5'-TTGGACGTGAGGCTTCCTG-3' (E2F4); 5'-TGCAAGGTCCGCTTCACCAG-3', 5'-TGCAAGGTCCGC TTCACCAG-3' (Zbtb7). Real-time PCR parameters used were as follows: $95^{\circ} \mathrm{C}$ for $10 \mathrm{sec} ; 95^{\circ} \mathrm{C}$ for $5 \mathrm{sec}, 60^{\circ} \mathrm{C}$ for $34 \mathrm{sec}$ for 40 cycles; $95^{\circ} \mathrm{C}$ for $15 \mathrm{sec}, 60^{\circ} \mathrm{C}$ for $1 \mathrm{~min}$ and $95^{\circ} \mathrm{C}$ for $15 \mathrm{sec}$.

Western blot analysis. HepG2 cells transfected with Zbtb7-targeting siRNA and scramble RNA were lysed on ice for $30 \mathrm{~min}$ with lysis buffer [10 $\mathrm{mM} \mathrm{N}$-(2-hydroxyethyl) piperazine-N'-2-ethane sulfonic acid, $10 \mathrm{mM} \mathrm{KCl}, 1 \mathrm{mM}$ ethylenediaminetetraacetic acid (EDTA) (pH 8.0), 0.1\% NP-40, $1 \mathrm{mM}$ DTT, $1 \mathrm{mM}$ PMSF and $0.5 \mathrm{mM} \mathrm{Na}_{3} \mathrm{VO}_{4}$ ). Soluble protein $(30 \mu \mathrm{g})$ was separated on a $12 \%$ sodium dodecyl sulfate (SDS)-polyacrylamide electrophoresis gel and blotted onto a pure nitrocellulose membrane (Bio-Rad, Hercules, CA) at $180 \mathrm{~mA}$ for $2 \mathrm{~h}$. Following blockage with 5\% skim milk in phosphate-buffered saline at room temperature for $45 \mathrm{~min}$, membranes were incubated with the Zbtb7 antibody (1:500; Sigma, St. Louis, MO, USA) and the CDK2 antibody (1:300; 
Abcam) in the same buffer for $2 \mathrm{~h}$, followed by incubation with goat anti-rabbit $\operatorname{IgG}$ (1:2000) for $1 \mathrm{~h}$. Antibody binding was detected using an enhanced chemiluminescence system (Pierce, Rockford, IL). To correct protein loading amounts, the membranes were reprobed with $\beta$-actin monoclonal antibody (1:40,000) (Sigma).

Cell cycle analysis. For cell cycle analysis, at $48 \mathrm{~h}$ following siRNA transfection, cells were harvested, washed with PBS and fixed in $70 \%$ chilled ethanol at $4^{\circ} \mathrm{C}$ for $30 \mathrm{~min}$, then treated with $1 \mathrm{mg} / \mathrm{ml}$ of RNase A (Sigma-Aldrich) and stained with $100 \mu \mathrm{g} / \mathrm{ml}$ of propidium iodide for $30 \mathrm{~min}$. The DNA content was determined with a fluorescent-activated cell sorter FACScan (Beckman Coulter, Inc, Miami, FL, USA). The data were analysed with cell cycle analysis software ModFit LT 2.0 (Verity Software House, Topsham, ME, USA). Most of the experiments were performed in triplicate.

Statistical analysis. Statistical analysis was performed using the Student's t-test with INSTAT statistical analysis package (Graph Pad Software). Significance was defined as $\mathrm{p}<0.05$.

\section{Results}

Zbtb7 downregulates the expression of CDK2 and E2F4 in hepatocarcinoma cells. To determine whether CDK2 and E2F4 are the potential downstream targets of the Zbtb7 gene in liver cancer cells, HepG2 and QGY7703 cells, two liver cancer cell lines, were utilized as a cell model. As shown in Fig. 1A and $\mathrm{B}$, the overexpression of $\mathrm{Zbtb} 7$ resulted in downregulation of CDK2 and E2F4 mRNA levels in both HepG2 and QGY7703 cells. To further confirm the observation at the mRNA level, Zbtb7-targeting siRNA was used to knock down the expression of Zbtb7 in HepG2 cells, a cell line with high endogenous Zbtb7 expression. As shown in Fig. 1C, the Zbtb7targeting siRNA-induced downregulation of $\mathrm{Zbtb} 7$ protein level led to an increase in CDK2 and E2F4 protein levels in the HepG2 cells. These observations suggest that CDK2 and E2F4 may be the downstream targets of Zbtb7 and that Zbtb7 functions as a negative modulator of CDK2 and E2F4 gene expression in liver cancer cells.

Zbtb7 represses the promoter activity of CDK2 and E2F4 genes in hepatocarcinoma cells. To elucidate the mechanism of Zbtb7-induced downregulation of the CDK2 and E2F4 genes, the core promoter sequences of the CDK2 and E2F4 genes were cloned and ligated to luciferase reporter plasmids (Fig. 2A and B). The delivery of the Zbtb7 expression plasmid into HepG2 and QGY7703 cells obviously repressed the promoter activities of the CDK2 and E2F4 genes (Fig. 2C and D) which indicates that $\mathrm{Zbtb} 7$ downregulated the expression of CDK 2 and E2F4 by suppressing the promoter activities in liver cancer cells. To further investigate whether $\mathrm{Zbtb} 7$ represses the promoter activities of CDK2 and E2F4 genes by a direct mechanism, ChIP PCR was carried out in the HepG2 cells. As observed in Fig. 2E, Zbtb7 was shown to bind with the core promoter sequences of the CDK2 and E2F4 genes. Those results indicate that $\mathrm{Zbtb} 7$ downregulated the expression of CDK2 and E2F4 genes by repressing the promoter activities by a direct mechanism that involved the direct binding of
A

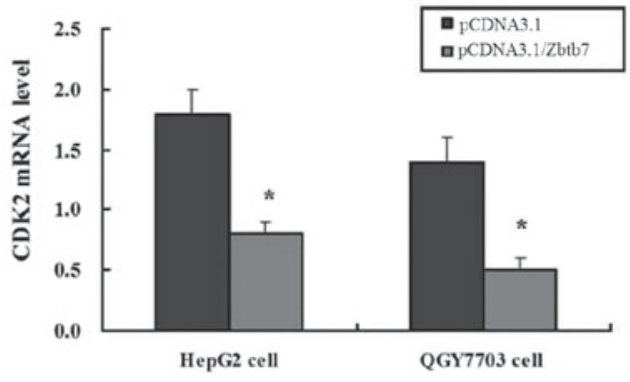

B

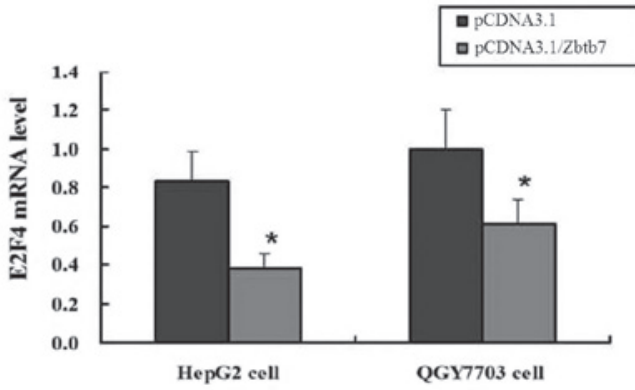

C

Zbtb7 siRNA Scramble RNA

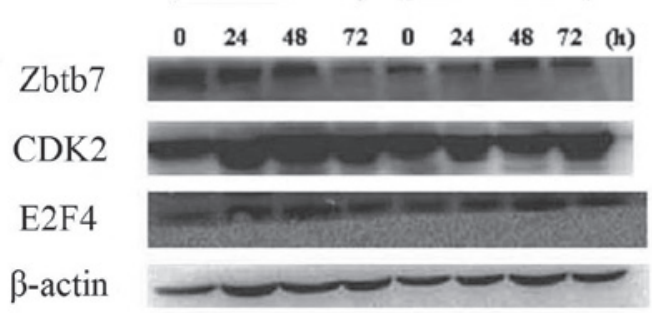

Figure 1. Zbtb7 suppresses the expression of CDK2 and E2F4 genes in hepatocarcinoma cells. (A and B) Zbtb7-expressing plasmids were transiently transfected into both HepG2 and QGY7703 cells, and at $48 \mathrm{~h}$ following transfection, RNA was extracted from the indicated cells and subjected to real-time PCR analysis to monitor the mRNA level of the CDK2 and E2F4 genes. The values are expressed as means \pm SD from three independent experiments. ${ }^{*} \mathrm{p}<0.05$. (C) Zbtb7-targeting siRNA was introduced into HepG2 cells, and cells were collected at indicated time points. The protein levels of Zbtb7, CDK2 and E2F4 were detected by western blot analysis.

Zbtb7 to the corresponding promoter sequences in the liver cancer cells.

Zbtb7 suppresses CDK2 promoter activity via an element at -259 to -249 . To determine the $\mathrm{Zbtb} 7$ regulatory elements within the CDK2 promoter sequence, site-directed mutagenesis was performed. The consensus Zbtb7 regulatory elements were identified as either a single guanine-rich site or a site with the consensus sequence $\mathrm{G}(\mathrm{A} / \mathrm{G}) \mathrm{GGG}(\mathrm{T} / \mathrm{C})(\mathrm{C} / \mathrm{T})(\mathrm{T} / \mathrm{C})(\mathrm{C} / \mathrm{T})$ (11). Searching for the $\mathrm{Zbtb} 7$ regulatory consensus sequences resulted in identification of three potential elements within the CDK2 promoter: -329 to $-322,-259$ to -252 , and -227 to -218 bp. As shown Fig. 3A, those sites were replaced with $G$ or $\mathrm{T}$ by mutagenesis. It was found that the mutation at -259 to -249 (PMS2, PDS2) restored Zbtb7-induced repression of CDK2 promoter activity in both HepG2 and QGY7703 cells, while no such observation was obtained when mutation occurred at the sites -329 to -322 and -227 to -218 (Fig. 3B and C). These results suggest that the site at -259 to -249 within the 
A

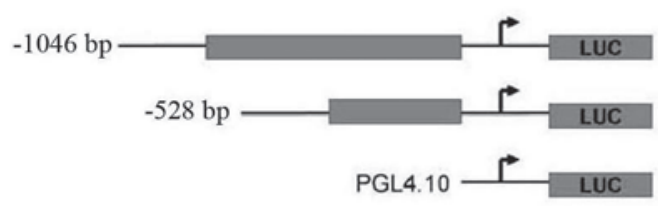

C

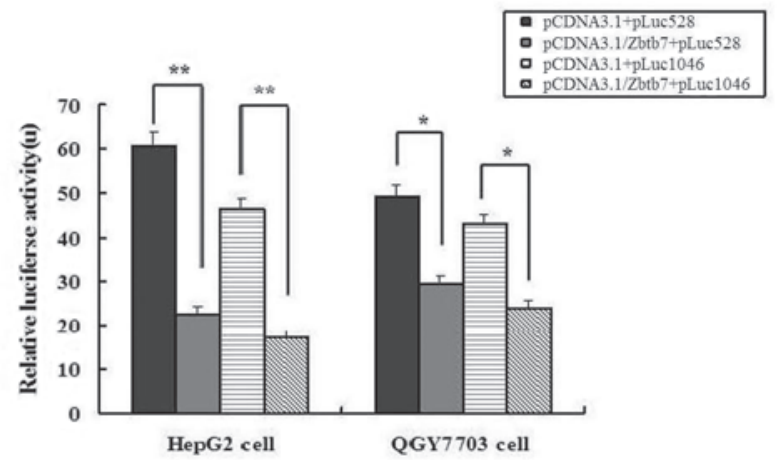

B

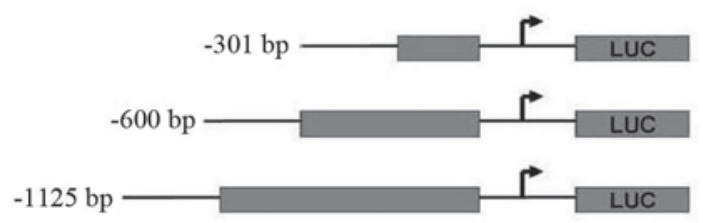

D

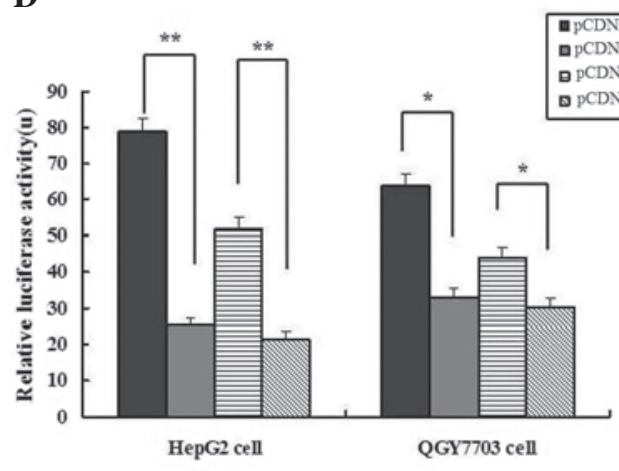

E

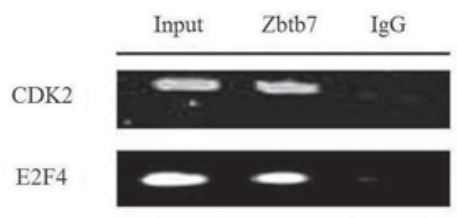

Figure 2. Zbtb7 represses the promoter activities of the CDK2 and E2F4 genes in hepatocarcinoma cells. (A and B) Schematic representation of CDK2 and E2F4 promoter luciferase reporter plasmids: pLuc-1046, pLuc-528, pLuc-1152, pLuc-600 and pLuc-301, containing a series of 5'-3' deleted promoters; (C and D) HepG2 and QGY7703 cells were transfected with promoter luciferase report plasmids and PCDNA3.1 or PCDNA3.1/Zbtb7, and cells were harvested for lucifrease activity assay as described in the text at $48 \mathrm{~h}$ following transfection. The values are expressed as the means \pm SD from three independent experiments. ${ }^{*} \mathrm{p}<0.05 ;{ }^{* *} \mathrm{p}<0.01$. (E) Sheared chromatin from HepG2 cells was immunoprecipitated using Zbtb7 antibody and ChIP-PCR was performed using primers targeting CDK2 (+1 to $-528 \mathrm{bp})$ and E2F4 (+1 to $-301 \mathrm{bp})$ promoter, respectively.

CDK2 promoter is responsible for Zbtb7-induced repression of promoter activity.

Zbtb7 induces G1/S arrest in hepatocarcinoma cells. Since CDK2 acts as a regulator of G1/S in cell cycle progression, it may be postulated that Zbtb7 might affect cell cycle distribution. To test this hypothesis, HepG2 and QGY7703 cells were treated with $50 \mathrm{nM}$ Zbtb7 siRNA for $48 \mathrm{~h}$, and the cell cycle distribution was analyzed by using a FACScan flow cytometer. Following incubation with $50 \mathrm{nM}$ Zbtb7 siRNA for $48 \mathrm{~h}$, $61.08 \%$ of HepG2 cells and $53.12 \%$ of QGY7703 cells were in $\mathrm{G} 0 / \mathrm{G} 1$ phase compared to $65.06 \%$ of the scramble siRNAtreated HepG2 cells and $68.32 \%$ of the scramble siRNA-treated QGY7703 cells were in the G0/G1 phase (Fig. 4A and B). These results suggest a role of $\mathrm{Zbtb} 7$ in the regulation of the $\mathrm{G} 1 / \mathrm{S}$ phase of the cell cycle in liver cancer cells.

\section{Discussion}

CDK2 was thought to be a regulator of G1/S transition in cell cycle progression (13), and its activity is regulated positively through an association with cyclins and negatively by CDK2 inhibitors. The TATA-less CDK2 promoter has been studied in NIH3T3 mouse fibroblasts (12) and multiple transcription factor binding sites have been identified. It was identified that the sequence spanning -100 to +108 is sufficient for basal promoter activity in NIH3T3 cells, specifically attributed to two SP1 sites within this region (12). In addition, a serum-responsive cis-element was found to be present 700 bp upstream of the transcriptional start. It was revealed that the transcriptional start site of the melanocyte-specific gene SILV/PMEL17 resides just 796 nucleotides away from the translational start site of the CDK2 gene (14), posing an interesting regulatory challenge.

In the present study, it was demonstrated that Zbtb7 acts as a negative regulator of CDK2 gene expression. Zbtb7-induced downregulation of CDK2 was confirmed at both the mRNA and protein level by gene delivery and siRNA transfection in HepG2 and QGY2203 cells. To further elucidate the mechanism underlying Zbtb7-induced downregulation of CDK2, the core promoter sequences of the CDK2 gene were cloned from HepG2 cells and ligated to luciferase reporter plasmids. A luciferase activity assay showed that the expression of Zbtb7 significantly repressed promoter activity of the CDK2 gene 
A

PMS1: 5'-GCCTGGGGCGGGATCATTTGC-3 5'-GCCTAAAAAAAGATCATTTGC-3'

PMS2: 5'-GGCCTGGGGGCGGAGCCAGGG-3' 5'-GGCCTTTTTTTTTAGCCAGGG-3'
PMS3: 5'-AGCAGGGGCGGGGCCTCTGGTG-3

5'-AGCATTTTTTTTTTCCTCTGGTG-3

PDS2: 5'-GGCCT.........AGCCAGGG-3'
B

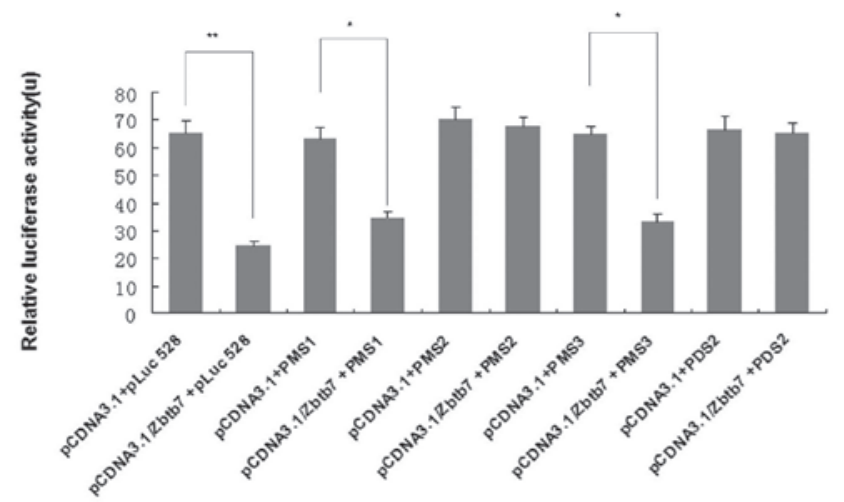

C

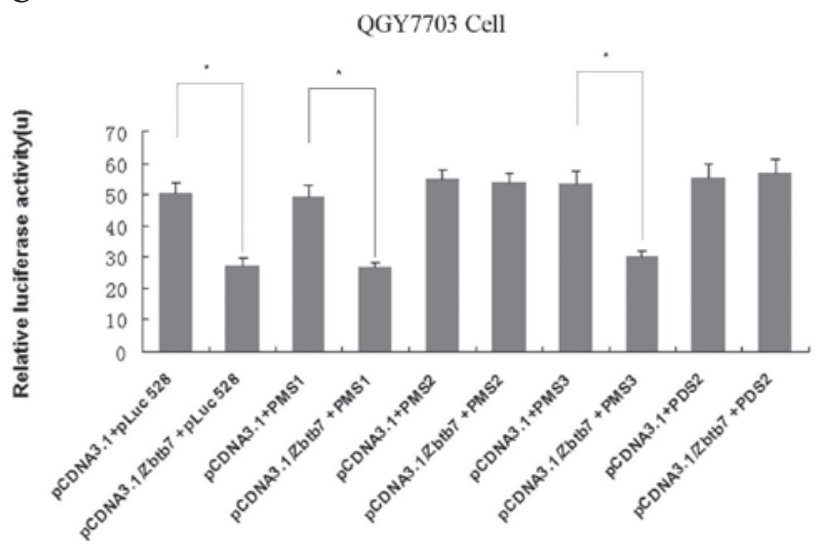

Figure 3. Zbtb7 suppresses CDK2 promoter activity via an element at -259 to -252. (A) Schematic representation of CDK2 promoter sequences for site-directed mutagenesis. (B and C) HepG2 and QGY7703 cells were transfected with the indicated promoter luciferase reporter plasmids and PCDNA3.1 or PCDNA3.1/ $\mathrm{Zbtb} 7$, and the cells were harvested for luciferase activity assay as described in the text at $48 \mathrm{~h}$ after transfection. The values are expressed as the means $\pm \mathrm{SD}$ from three independent observations.. $\mathrm{p}<0.05,{ }^{* *} \mathrm{p}<0.01$.

A
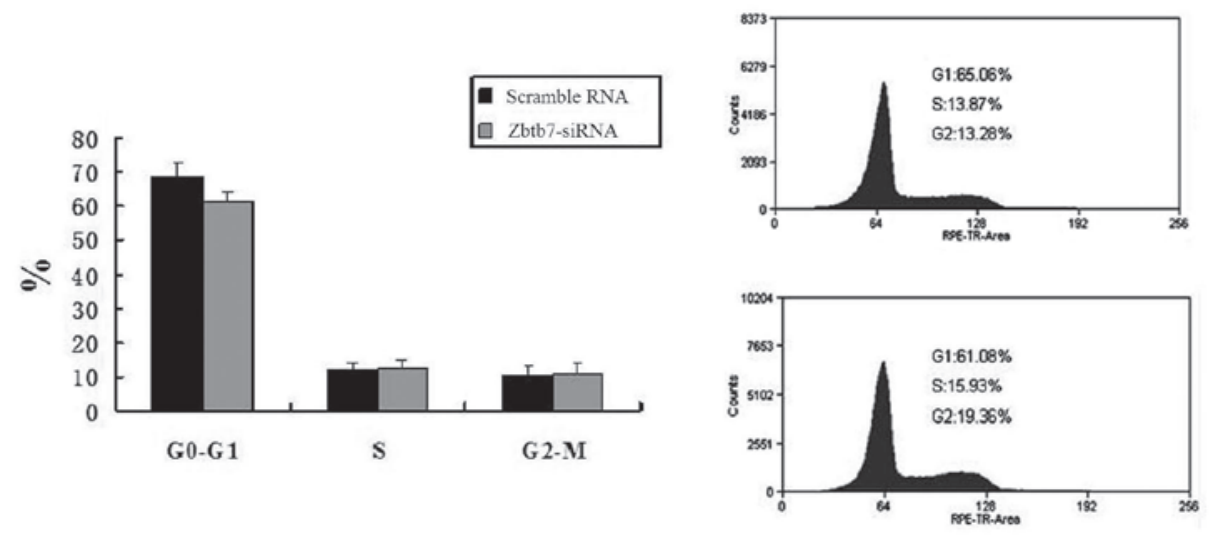

Zbtb7 siRNA

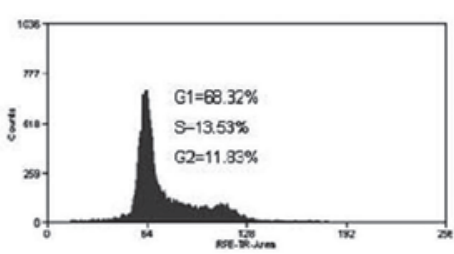

Scramble RNA

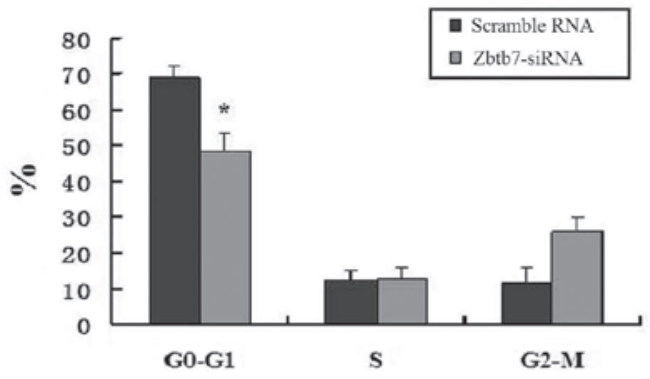

Scramble RNA

\section{B}

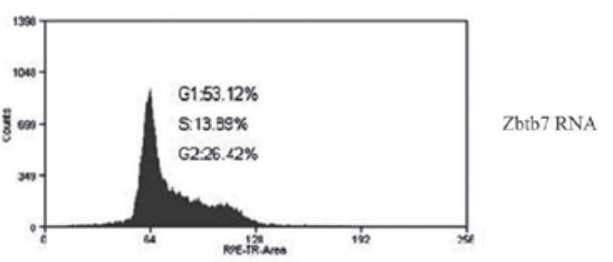

Figure 4. Zbtb7 induces G1/S arrest in hepatocarcinoma cells. (A) HepG2 cells were treated with Zbtb7-targeting siRNA or scamble siRNA for 48 h, and cell cycle analysis was performed with a FACscan flow cytometer. (B) QGY7703 cells were treated with Zbtb7-targeting siRNA or scamble siRNA for $48 \mathrm{~h}$, and the cells were harvested for cell cycle analysis. Right panels: measurements using a FACScan flow cytometer. Left panels: quantification of cell cycle distribution from the right panels. ${ }^{*} \mathrm{p}<0.05$. 
in both HepG2 and QGY2203 cells. ChIP PCR revealed that Zbtb7 suppressed promoter activity of the CDK2 gene through its binding with the core promoter sequences. To further determine the cis elements within the core promoter sequences of the CDK2 gene which are responsible for Zbtb7-induced repression of promoter activity, three potential Zbtb7 binding elements were utilized for mutagenesis analysis. The mutation or deletion at -259 to -249 (PMS2, PDS2) of the CDK2 promoter resulted in diminished Zbtb7-induced repression of CDK2 promoter activity, which suggests that the site at -259 to -249 within the CDK2 promoter is responsible for Zbtb7-induced repression of promoter activity.

E2F4, one of the E2F family, has been implicated in cell cycle regulation, and its deregulation can lead to neoplastic transformation of certain immortalized cell lines (15-18). E2F4 was identified as a downstream target of Zbtb7, and it was shown that Zbtb7-induced downregulation of E2F4 results in promotion of terminal preadipocyte differentiation (8). We also demonstrated Zbtb7 to be a negative regulator of E2F4 expression in two liver cancer cell lines, HepG2 and QGY7703 cells. Zbtb7 was shown to repress E2F4 promoter activity through direct binding with the promoter sequence in human liver cancer cells which is consistent with the observation in preadipocytes (8).

In the present study, we provide evidence that Zbtb7 acts as a negative regulator of both CDK2 and E2F4 expression by a direct mechanism that involves binding of Zbtb7 with the promoter sequences in liver cancer cells. CDK2 and E2F4 were shown to be regulators of the G1/S phase in cell cycle progression, so it can be postulated that Zbtb7 might have a role in cell cycle regulation. To test this hypothesis, cell cycle analysis was performed in HepG2 and QGY7703 cells. Notably, it was found that the siRNA-induced Zbtb7 knockdown led to a lower percentage of cells in G1 phase which suggests a negative role for Zbtb7 in the regulation of G1/S entry in liver cancer cells and the repression of CDK2 and E2F4 induced by Zbtb7 might explain our observation. The mechanism underlying Zbtb7-induced cell cycle arrest at G1/S remains to be further characterized.

In conclusion, in the present study CDK2 and E2F4 were identified as downstream targets of Zbtb7 in liver cancer cells. Zbtb7 was shown to negatively regulate the expression of both CDK2 and E2F4 genes through a mechanism involving the direct binding of Zbtb7 to promoter sequences. The evidence for the role of Zbtb7 in the regulation of cell cycle progression was also provided, and our finding might have important implications for understanding the role of Zbtb7 in carcinogenesis, development and differentiation.

\section{Acknowledgements}

This study was supported by a grant from the National 863 Project of the Ministry of Science and Technology, China (2007AA02Z160) and by a grant from the China Postdoctoral Foundation (200880440390).

\section{References}

1. Davies JM, Hawe N, Kabarowski J, et al: Novel BTB/POZ domain zinc-finger protein, LRF, is a potential target of the LAZ-3/BCL-6 oncogene. Oncogene 18: 365-375, 1999.

2. Maeda T, Hobbs RM, Merghoub T, Guernah I, Zelent A, Cordon-Cardo C, Teruya-Feldstein J and Pandolfi PP: Role of the proto-oncogene Pokemon in cellular transformation and ARF repression. Nature 433: 278-285, 2005.

3. Kukita A, Kukita T, Ouchida M, Maeda H, Yatsuki H and Kohashi O: Osteoclast-derived zinc finger (OCZF) protein with POZ domain, a possible transcriptional repressor, is involved in osteoclastogenesis. Blood 94: 1987-1997, 1999.

4. Morrison DJ, Pendergrast PS, Stavropoulos P, Colmenares SU, Kobayashi R and Hernandez N: FBI-1, a factor that binds to the HIV-1 inducer of short transcripts (IST), is a POZ domain protein. Nucleic Acids Res 27: 1251-1262, 1999.

5. Lee DK, Suh D, Edenberg HJ and Hur MW: POZ Domain transcription factor, FBI-1, represses transcription of $\mathrm{ADH} 5 / \mathrm{FDH}$ by interacting with the zinc finger and interfering with DNA binding activity of Sp1. J Biol Chem 277: 26761-26768, 2002.

6. Apostolopoulou K, Pateras IS, Evangelou K, Tsantoulis PK, Liontos M, Kittas C, Tiniakos DG, Kotsinas A, Cordon-Cardo C and Gorgoulis VG: Gene amplification is a relatively frequent event leading to ZBTB7A (Pokemon) overexpression in non-small cell lung cancer. J Pathol 213: 294-302, 2007.

7. Maeda T, Merghoub T, Hobbs RM, Dong L, Maeda M, Zakrzewski J, van den Brink MR, Zelent A, Shigematsu H, Akashi K, Teruya-Feldstein J, Cattoretti G and Pandolfi PP: Regulation of B versus T lymphoid lineage fate decision by the proto-oncogene LRF. Science 316: 860-866, 2007.

8. Laudes M, Bilkovski R, Oberhauser F, Droste A, Gomolka M, Leeser U, Udelhoven $M$ and Krone W: Transcription factor FBI-1 acts as a dual regulator in adipogenesis by coordinated regulation of cyclin-A and E2F-4. J Mol Med 86: 597-608, 2008.

9. Choi WI, Jeon BN, Park H, Yoo JY, Kim YS, Koh DI, Kim MH, Kim YR, Lee CE, Kim KS, Osborne TF and Hur MW: Protooncogene FBI-1 (Pokemon) and SREBP-1 synergistically activate transcription of fatty-acid synthase gene (FASN). J Biol Chem 832: 29341-29354, 2008.

10. Jeon BN, Yoo JY, Choi W, Lee CE, Yoon HG and Hur MW: Protooncogene FBI-1 (Pokemon/ZBTB7A) represses transcription of the tumor suppressor $\mathrm{Rb}$ Gene via binding competition with Sp1 and recruitment of co-repressors. J Biol Chem 283: 33199-33210, 2008.

11. Pessler F and Hernandez N: Flexible DNA Binding of the BTB/ POZ-domain Protein FBI-1. J Biol Chem 278: 29327-29335, 2003.

12. Shiffman D, Brooks EE, Brooks AR, Chan CS and Milner PG: Characterization of the human cyclin-dependent kinase 2 gene. Promoter analysis and gene structure. J Biol Chem 271: 12199-12204, 1996.

13. Ekholm SV and Reed SI: Regulation of G(1) cyclin-dependent kinases in the mammalian cell cycle. Curr Opin Cell Biol 12: 676-684, 2002.

14. Adachi N and Lieber MR: Bidirectional gene organization: a common architectural feature of the human genome. Cell 109: 807-809, 2002.

15. Ginsberg D, Vairo G, Chittenden T, Xiao Z-X, Xu G, Wydner KL, DeCaprio JA, Lawrence JB and Livingston MD: E2F-4, a new member of the E2F transcription factor family, interacts with p107. Genes Dev 8: 2665-2679, 1994.

16. Beijersbergen RL, Kerkhoven RM,Zhu L, Carlee L,Voorhoeve PM and Bernards R: E2F-4, a new member of the E2F gene family, has oncogenic activity and associates with p107 in vivo. Genes Dev 8: 2680-2690, 1994.

17. Singh P, Wong SH and Hong W: Overexpression of E2F-1 in rat embryo fibroblasts leads to neoplastic transformation. EMBO J 13: 3329-3338, 1994

18. Xu G, Livingston DM and Krek W: Multiple members of the E2F transcription factor family are the products of oncogenes. Proc Natl Acad Sci USA 92: 1357-1361, 1995. 